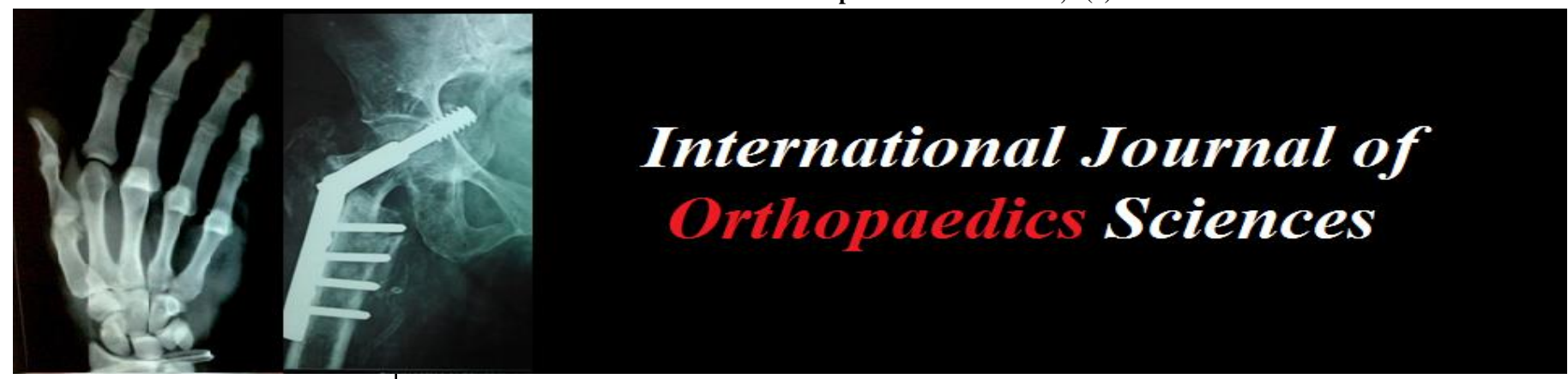

E-ISSN: 2395-1958

P-ISSN: 2706-6630

IJOS 2020; 6(3): 909-911

(C) 2020 IJOS

www.orthopaper.com

Received: 14-07-2020

Accepted: 08-08-2020

Dr. Giddi Rajani Kumar M.S Orthopaedics, Assistant Professor Guntur medical college, Guntur, Andhra

Pradesh, India
Corresponding Author: Dr. Giddi Rajani Kumar M.S Orthopaedics, Assistant Professor Guntur medical college, Guntur, Andhra Pradesh, India

\section{Neglected anterior shoulder dislocation managed by open reduction combined with Latarjet procedure}

\section{Dr. Giddi Rajani Kumar}

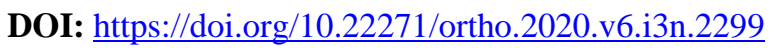

\section{Abstract}

Introduction: Neglected shoulder dislocation may be accompanied by pathological changes in bony and soft tissue structure. Therefore, requires extensive surgical procedure. There is no standard treatment protocol to overcome this case and it is still a challenging case.

Presentation of case: We presented a 46-year-old male patient with deformity on his left shoulder. The patient has neglected anterior shoulder dislocation from 6 month with a Hill-Sachs lesion. Treatment was open reduction combined with Latarjet procedure. Evaluation of treatment was performed followed by surgery.

Discussion: Neglected shoulder dislocation, especially with significant bony defects, is challenging and not be managed with standard surgical technique because of severe soft-tissue contracture and bone deficiency. Chronic anterior shoulder dislocation is a difficult condition for both patient and clinician. In addition, the treatment results can be unsatisfactory. Latarjet procedure has been shown to be effective for the treatment of recurrent anterior shoulder dislocation with a large glenoid defect which might justify the application of this procedure for the treatment of neglected anterior shoulder dislocation.

Conclusion: Open Latarjet procedure performed for treatment of neglected anterior shoulder dislocation have a high rate of successful in preventing further dislocation of the shoulder joint although the high risk of osteoarthritis of the shoulder joint can still persist.

Keywords: Shoulder immobilisation, Latarjet procedure, anterior shoulder dislocation, Bankart's lesion

\section{Introduction}

Shoulder joint is the most common dislocated joint. Anterior dislocation of shoulder occurs more frequent, it accounts for $95 \%$ of all shoulder dislocation, rather than posterior dislocation. A neglected shoulder dislocation is rare and can be accompanied by pathological changes in bony and soft tissue structures. Therefore, it requires extensive surgical procedure. Until now no standard treatment for this case and it is a difficult problem for both patients and clinicians. We present a 46-year-old male who came to our outpatient department in Guntur medical college and hospital, Guntur, has suffered neglected anterior dislocation for six months with a Hill-Sachs lesion. We managed this case by open reduction and Latarjet procedure.

This report is based on consensus-based surgical case report guidelines, SCARE criteria

\section{Case Discussion}

A 46-year-old male presented with a chief complaint of deformity on his left shoulder for six months before hospital admission in October 2015 [Fig 1]. The patient had a slip in the bathroom and fell in sitting position with left arm supporting the body. After the accident, the left shoulder was painful and looked deformed. Then the patient went for native treatment and was massaged and splinted, but the shoulder was still painful and looked deformed. The patient used an arm sling to immobilize his left shoulder for about six months. Gradually, patient could do his normal daily activity with limited movement of left shoulder.

The patient complained of limited movement of his left shoulder with some pain. On physical examination we found deformity on the left shoulder, like squaring of shoulder, and muscle atrophy. Neurovascular examination was normal. The range of motion (ROM) of left shoulder was extension-flexion $20^{\circ}-90^{\circ}$, abduction-adduction $20^{\circ}-70^{\circ}$, internal-external rotation 
$30^{\circ}-30^{\circ}$. The antero-posterior X-ray imaging showed anterior dislocation of left glenohumeral joint and Computed Tomography (CT) scan showed a Hill-Sachs lesion on the humeral head. We diagnosed the patient had a neglected anterior shoulder dislocation with a Hill-Sachs lesion and performed an open reduction and Latarjet procedure to treat this patient.

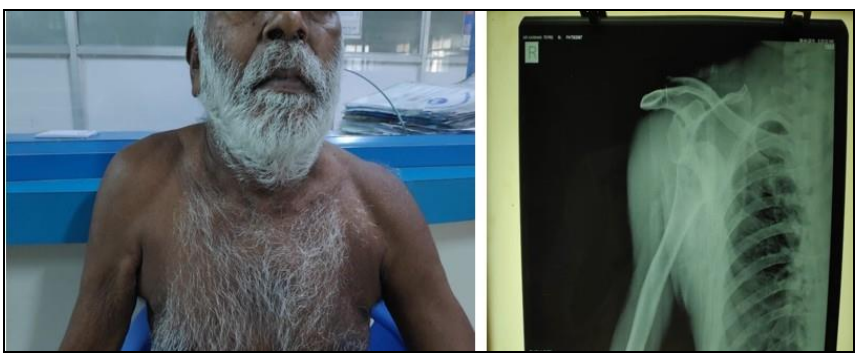

Fig 1: Six months after the accident, patient decided to seek medical help to treat his left shoulder.

We performed an open reduction surgery using anterior approach of shoulder [Fig 2] and found massive fibrotic tissue around the joint and the Hill-Sachs lesion. We removed all the fibrotic tissue to create the space for shoulder joint to be reduced. After reducing the dislocation, we inserted a Kirschner wire to add stability for maintaining the reduced shoulder, then continued on Latarjet procedure. The Latarjet procedure was performed by cutting the coracoid process and transferred it with conjoint tendon to antero-inferior part of glenoid and fixed by two screws.
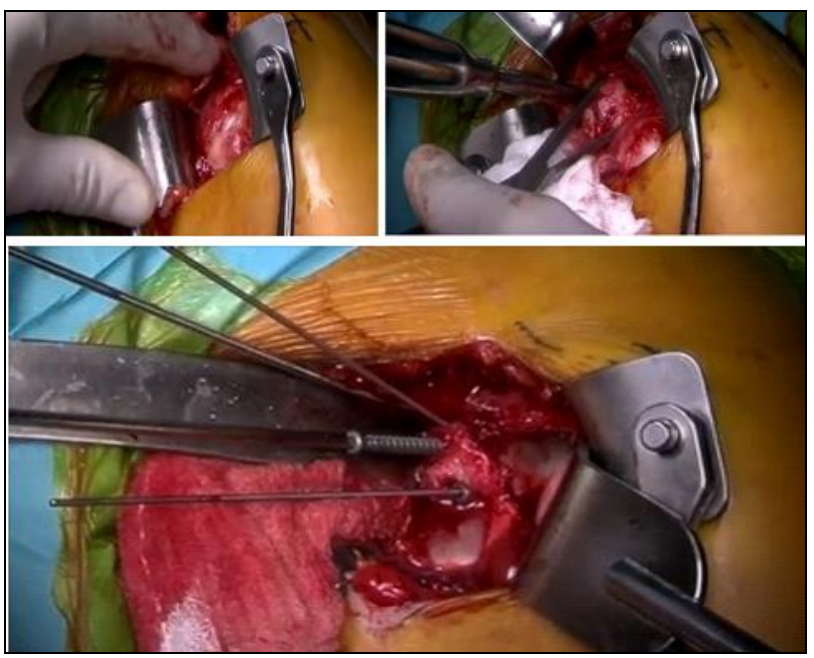

Fig 2: open reduction surgery using anterior approach of shoulder

The final result showed that the glenohumeral joint has been reduced with wire fixation. Post-operative X-ray showed a reduced shoulder joint. Unfortunately, there was a claw hand on his left hand due to a neuropraxia of the ulna. The patient was discharged 2 days after surgery. We removed the $\mathrm{K}$-wire after 3 weeks, then the patient started the rehabilitation program. The patient also underwent Transcutaneous Electrical Nerve Stimulation (TENS) and range of motion exercises. We evaluated the patient for 3 months in outpatient clinic. Three months after surgery, the ulnar neuropraxia was healed. The patient still had a limited ROM, (abduction $0^{\circ}-$ $100^{\circ}$ ) on his left shoulder. At ten months follow-up, the patient had no recurrent dislocation.

\section{Discussion}

The glenohumeral joint is the most frequently dislocated joint in the body. Anterior dislocation of shoulder occurs more frequent and accounts for $95 \%$ of all shoulder dislocation ${ }^{[3]}$. The most common mechanism for the unilateral injuries is trauma. A traumatic event could lead to anterior shoulder dislocation when it was happened in abducted and extended arm position so greater tuberosity abuts against acromion, causing leverage forces leading humeral head to come out of glenoid cavity. The term of chronic dislocation of the shoulder is applied to condition where there is loss of recognition of injury for at least 3 or 4 weeks, although other authors have described chronic dislocation with various duration. A neglected shoulder dislocation may be accompanied with pathological changes in bony and soft tissue structures. Therefore, it requires an extensive surgical procedure.

A neglected shoulder dislocation, especially with significant bony defects, is a dilemmatic condition since it cannot be managed by standard surgical procedure and concomitant lesions are common, including Hill-Sachs and Bankart lesions, massive glenoid bone loss, rotator cuff tear; and later severe glenohumeral osteoarthritis could also occur. Because of severe soft-tissue contracture and imbalance as well as bone deficiency, neglected anterior shoulder dislocation is a difficult problem for both patients and clinicians.

The outcomes of some procedures, such as Bankart repair, remplissage, coracoid transfer, bone-grafting and arthroplasty, in restoring the stability of the shoulder were varied and the overall failure rates were quite high. The choice of treatment included observation, manipulation, open reduction with or without allograft reconstruction, Bankarts repair, capsulolabrial repair and arthroplasty ${ }^{[2,4]}$. Surgical treatment for chronic shoulder dislocation is usually advocated for better functional outcome, although the results may be poor and unsatisfactory. The open reduction surgery was mostly recommended if the dislocation has been neglected more than four weeks after injury, in order to reduce the risk of concomitant fracture or cartilage injury. Several surgical procedures have been reported a gleno-humeral transfixation by using smooth pins through the head into the glenoid for maintaining reduction. The acromio-humeral transfixing pins could halt joint motion for 3-4 weeks.

The neglected cases generally have significant bony defects due to constant friction of the dislocated humeral head against the anterior border of glenoid, which was also found in our patient ${ }^{[1,6]}$. The bony defect can cause recurrent instability but it depends on the size and depth of the defect. In defects more than $25 \%$ but less than $40 \%$, the anatomic procedures, such as allograft reconstruction of the head, humeral head disimpaction/humeroplasty and non-anatomic procedures, such as osseous or soft tissue (remplissage) transfer of the infraspinatus and Latarjet procedure, are recommended. Latarjet provides stability by its 'triple effect' and it is more familiar for the surgeon than remplissage procedure.

Latarjet procedure has been proven to be effective for the treatment of recurrent anterior shoulder dislocation with a large glenoid osseous defect which might justify the application of this procedure for the treatment for neglected anterior shoulder dislocation [2]. Transfer of osteotomized coracoid process into the glenoid rim was described by Latarjet in 1958. The transfer includes a portion of coracoacromial ligament which is sutured to the anterior capsule through a short horizontal incision in subscapularis. Latarjet procedure reconstructs the depth and width of the glenoid. A dynamic reinforcement is created for inferior part of the capsule through the coracobrachialis muscle, which is 
particularly effective when the arm is abducted and externally rotated. Burkhart et al., as cited by An et al, reported excellent outcome of Latarjet procedure in 102 patients, who either had more than $25 \%$ of glenoid bone loss or an engaging Hill-Sachs lesion, with only $4.9 \%$ recurrence rate after a mean follow-up of 59 months ${ }^{[5]}$. In defects that comprise more than $40 \%-50 \%$ of the head, rotational proximal humeral osteotomy in young patients and partial or total humeral head arthroplasty are recommended. It has been suggested that, compared with soft-tissue reconstruction, such as Bankart repair, an open Latarjet procedure is more effective for the treatment of recurrent anterior shoulder dislocation with a marked glenoid osseous defect. Nevertheless, it was reported a high rate of redislocation or subluxation, loss of external rotation and internal rotation, and the deterioration or early onset of glenohumeral osteoarthritis after the Latarjet procedure. In our case, the patient still had limited ROM, $100^{\circ}$ of abduction after Latarjet procedure but no redislocation.

Nerve injury after the surgery is a common complication that can be happened during transferring the coracoid process or during surgical exploration to reduce the dislocation. The most common nerve injury is from musculocutaneous nerve and axillary nerve, but it can occur in any brachial plexus branches and mostly can be recovered spontaneously. In this patient, the ulnar nerve neuropraxia happened during the exploration process to reduce the dislocation.

Soft-tissue imbalance is another risk factor for postoperative redislocation or subluxation. In patients with neglected anterior shoulder dislocation, the long-term may cause the lengthening and thinning of the musculotendinous unit or changing the balance of the glenohumeral joint ${ }^{[3,8]}$. A high rate of glenohumeral osteoarthritis deterioration also noted. Postoperative shoulder osteoarthritis is one of the complications that can occur due to avascular necrosis of the humeral head. There are many factors that contributing to avascular necrosis that leads to shoulder osteoarthritis for examples increased age at the time of first dislocation, increased age at the time of surgery, and presence of arthritis before surgery; however, there is no specific time when the avascular necrosis starting to occur. Shoulder osteoarthritis can be occurred as a result of preexisting chondral injury, which leads to degeneration over time, or as a result of the operation procedure. Despite the risk of avascular necrosis of humeral head in the long term follow up, Can be consider for the Latarjet procedure performed in this patient has successfully stabilized the shoulder joint of the neglected dislocation.

This case report has a limitation that the follow-up period to analyze the stability of the shoulder was only 3 months after the surgery. Another long term follow-up should be considered to evaluate the shoulder stability and other surgical complications.

\section{Conclusion}

In conclusion, open reduction combined with Latarjet procedure performed for treatment of neglected anterior shoulder dislocation was found to have a high rate of successful in preventing further dislocation of the shoulder joint although high risk of osteoarthritis of the shoulder joint can still persist.

\section{References}

1. Chaudhary D, Joshi D, Jain V, Mohindra M, Mehta N. A six months old neglected anterior shoulder dislocation managed by closed reduction and Laterjet procedure. Chin.

2. AN VVG, Sivakumar BS, Phan K, Trantalis JA. systematic review and meta-analysis of clinical and patient-reported outcomes following two procedures for recurrent traumatic anterior instability of the shoulder: Latarjet procedure vs. Bankart repair. J. Shoulder Elb. Surg. 2016; 25:853-863.

3. Poggetti A, Castellini I, Neri E, Marchettil S, Lisanti M. Atraumatic bilateral neglected anterior shoulder dislocation: case report of a Jehovah's Witness 28-yearold male affected by iron-deficiency anemia and treated with bilateral Latarjet procedure. J. Orthop. Case Rep. 2015; 5:81-83.

4. Ropars M, Cretual A, Kaila R, Bonan I, Hervé A, Thomazeau $\mathrm{H}$ et al. Diagnosis and treatment of anteroinferior capsular redundancy associated with anterior shoulder instability using an open Latarjet procedure and capsulorrhaphy. Knee Surg. Sports Traumatol. Arthrosc. 2016; 24:3756-3764.

5. Peshin C, Jangira V, Gupta RK, Jindal R. Neglected anterior dislocation of shoulder with large Hillsach's

6. Xu W, Huang L-X, Guo JJ, Jiang D-H, Zhang Y, Yang H-L et al. Neglected posterior dislocation of the shoulder: a systematic literature review. J. Orthop. Transl. 2015; 3:89-94.

7. Agha RA, Borelli MR, Farwana R, Koshy K, Fowler A, Orgill DP et al. For the SCARE Group The SCARE 2018 Statement: updating consensus surgical case report (SCARE) guidelines. Int. J Surg. 2018; 60:132-136.

8. Provencher MT, Frank RM, LeClere LE, Metzger PD, Ryu JJ, Bernhardson A et al. The Hill-Sachs lession: diagnosis, classification, and management. J. Am. Acad. Orthop. Surg. 2012; 20:242-252. 